\title{
Density measurements of well samples from the Clear Lake-Geysers area, Lake and Sonoma Counties, California
}

by

\author{
Carter Roberts ${ }^{1}$
}
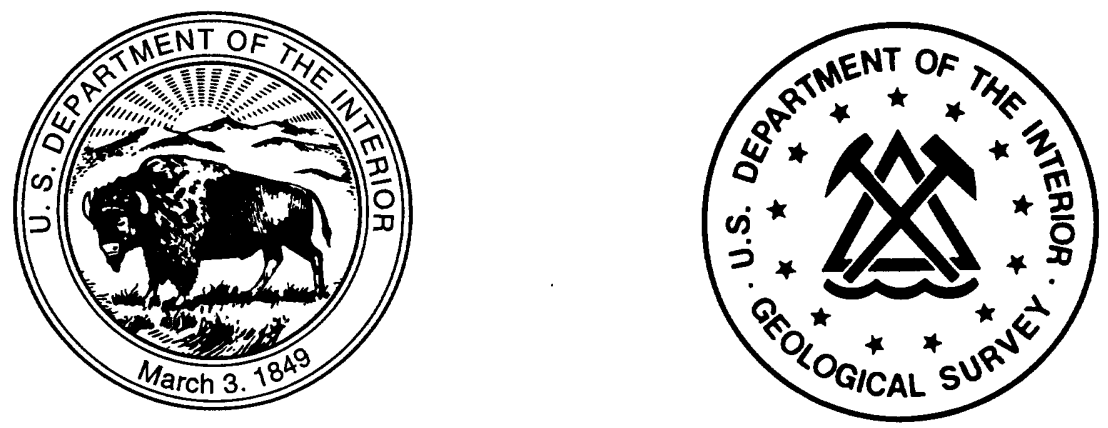

Open-File Report 97-427

1997

\begin{abstract}
This report is preliminary and has not been reviewed for conformity with U.S. Geological Survey editorial standards or the North American Stratigraphic Code. Any use of trade or form names is for descriptive purposes only and does not imply endorsement by the U.S. Geological Survey.
\end{abstract}

${ }^{1}$ Menlo Park, CA 94025 


\section{INTRODUCTION AND PROCEDURES/METHODS}

To aid with geophysical modeling and interpretation, density values were determined of cuttings and a few larger pieces from five wells in an area southwest of Clear Lake.

The Occidental Geothermal Neasham No. 1 well (Table 1) is located south of Mt Konocti in the SW $1 / 4$ of the NW $1 / 4$ of the SW $1 / 4$ of Sec. 33, T13N, R8W.

The Magma Watson No. 1 well (Table 2) is located about $3 \mathrm{~km}$ southwest of Konocti Bay on the lower south slope of Mt Konocti in the SW 1/4 of the SE 1/4 of Sec. 20, T13N, R8W.

The Occidental Geothermal No. 68-21 well (Table 3) is located about $5 \mathrm{~km}$ east of The Geysers in Sec. 21, T11N, R8W.

The Boggs 77-1 well (Tables 4a and 4b) is located near Boggs Lake about midway between The Geysers and Clear Lake. Two samples of cuttings from it were measured and a few larger samples.

The Eureka-Magma-GRI-Getty Kettenhoffen No. 1 well (Table 5) is located about $2.5 \mathrm{~km}$ southwest of Konocti Bay, just southwest of the normally dry lake in Ely Flat and about 2400 feet north and 2250 feet west of the southeast corner of Sec. 28, T13N, R8W.

For all the wells, the identification on each sample bag was the depth range of the sample in feet. These identifications have been left in feet to avoid confusion. Since most sample bags contained very little material and it was desired that a portion of each sample be retained for future use, between two and five samples were combined to obtain enough material.

To measure the cuttings, small cylindrical containers with a volume of 8.725 $\mathrm{cm}^{3}$ were weighed, then filled with well cuttings from a particular zone within a well and weighed again. The containers were then placed within a vacuum chamber and the air evacuated. Water was admitted to the chamber and the samples were left overnight before each container was removed from the water, surface water was removed and the now-saturated sample was reweighed. Only 16 samples per day could be measured with this procedure so samples were selected at fairly uniform intervals over the ranges for which samples were available.

For each sample, the following information was known:

Empty weight of container (we)

Volume of container $8.725 \mathrm{~cm}^{3}$

Weight of container plus sample (dry) (wd)

Weight of container plus sample saturated with water (ww)

The density could then be calculated as follows:

Weight of sample $=$ wd - we

Volume of sample $=8.725-(\mathrm{ww}-\mathrm{wd})$

Therefore:

$$
\text { Density }=\frac{w d-w e}{8.725+w d-w w}
$$




\section{ACKNOWLEDGEMENTS}

Comments regarding the lithology and stratigraphic assignments of the samples are from lithologic logs and notes by Robert J. McLaughlin, B. Carter Hearn, Jr., H.N. Ohlin, Cynde Sears, and Julie Donnelly-Nolan, USGS.

Lithology of the Occidental Geothermal Inc. well \#68-21 is from the log by R.F. Smith Corp.

Lithology of the Neasham \#1 well is by Robert J. McLaughlin, B. Carter Hearn, Jr., H.N. Ohlin, Cynde Sears, and Julie Donnelly-Nolan, USGS.

I wish to thank Colin Williams and Richard Keating for providing access to and training in the use of the equipment that made it possible to obtain these measurements. 
Table 1. Density values for the Occidental Geothermal, Neasham \# 1 well, located in Sec. 33 T13N, R8W, Lake County, CA

\begin{tabular}{|c|c|c|}
\hline $\begin{array}{l}\text { Sample } \\
\text { Identification }\end{array}$ & $\begin{array}{l}\text { Density } \\
\mathrm{g} / \mathrm{cm}^{3}\end{array}$ & Lithologies \\
\hline $210-250$ & 2.524 & \multirow[t]{3}{*}{$\begin{array}{l}\text { Rhyodacite of Sulphur Mound Mine, crystal rich, } \\
\text { mostly light gray to dark gray, with intervals of } \\
\text { reddish altered rhyodacite, in part altered. }\end{array}$} \\
\hline $400-450$ & 2.450 & \\
\hline $600-650$ & 2.595 & \\
\hline $1000-1050$ & 2.530 & \multirow{6}{*}{$\begin{array}{l}\text { Rhyodacite of Sulphur Mound Mine?, crystal rich, } \\
\text { mostly light gray to dark gray, in part altered. }\end{array}$} \\
\hline $1200-1250$ & 2.607 & \\
\hline $1400-1450$ & 2.602 & \\
\hline $1600-1650$ & 2.716 & \\
\hline $1800-1850$ & 2.650 & \\
\hline $2000-2050$ & 2.740 & \\
\hline $2200-2250$ & 2.554 & $\begin{array}{l}\text { Rhyodacite of Diener Drive, phenocryst-poor, } \\
\text { contains tiny plagioclase laths, dark gray to black, } \\
\text { in part glassy. }\end{array}$ \\
\hline $2730-2780$ & 2.781 & \multirow[t]{3}{*}{ Silty argillite w/talc-serp, and db/gb pods } \\
\hline $3000-3050$ & 2.769 & \\
\hline $3400-3450$ & 2.779 & \\
\hline $3800-3850$ & 2.715 & Clayey gouge \\
\hline $4200-4250$ & 2.732 & \multirow[t]{2}{*}{ Silty argillite w/talc-serp } \\
\hline $4400-4450$ & 2.698 & \\
\hline $5500-5550$ & 2.784 & \multirow[t]{2}{*}{ Argillite and sandstone w/minor Sp/talc } \\
\hline $5800-5850$ & 2.824 & \\
\hline $6500-6550$ & 2.701 & \multirow[t]{3}{*}{ Argillite w/abundant Sp/talc lenses } \\
\hline $6600-6650$ & 2.842 & \\
\hline $6900-6950$ & 2.754 & \\
\hline $8600-8650$ & 2.928 & \multirow{2}{*}{$\begin{array}{l}\text { Argillite; Gabbro, diabase, subequal mixture, with } \\
\text { felsic intrusive rocks in upper part of interval }\end{array}$} \\
\hline $9000-9050$ & 2.870 & \\
\hline $9400-9450$ & 3.170 & Hornfelsic argillite; Gabbro, diabase \\
\hline $9750-9800$ & 3.422 & Hornfelsic argillite (tourmalinized) \\
\hline
\end{tabular}


Table 2. Eureka-Magma Inc., Watson \#1 SW corner SE 1/4 SE 1/4 Sec 20 T13NR8W

\begin{tabular}{|c|c|c|}
\hline \begin{tabular}{l|} 
Sample \\
Identification
\end{tabular} & $\begin{array}{l}\text { Density } \\
\mathrm{g} / \mathrm{cm}^{3}\end{array}$ & Lithologies \\
\hline $280-310$ & 2.637 & Dacite of Benson Ridge (biotite dacite) \\
\hline $360-390$ & 2.521 & \multirow[t]{3}{*}{ Dacite of Bell Mine (phenocryst-poor dacite) } \\
\hline $600-630$ & 2.626 & \\
\hline $820-850$ & 2.656 & \\
\hline $1060-1090$ & 2.554 & \multirow[t]{2}{*}{$\begin{array}{l}\text { Basin deposits (fine-grained clay-rich to } \\
\text { coarse sandstone and gravel, rich in dacite } \\
\text { fragments) }\end{array}$} \\
\hline $1200-1230$ & 2.596 & \\
\hline $1370-1400$ & 2.613 & $\begin{array}{l}\text { Dacite north of Ely Flat (biotite-hornblende } \\
\text { dacite, prev. older dacite of Mt Konocti or } \\
\text { dacite of Ely Ridge) }\end{array}$ \\
\hline $1580-1610$ & 2.591 & \multirow[t]{7}{*}{$\begin{array}{l}\text { Rhyolite west of Sugarloaf (biotite rhyolite, } \\
\text { crystal rich; prev. biotite ry. of Pink Hill, } \\
\text { "Honeycutt" ry) }\end{array}$} \\
\hline $2000-2030$ & 2.657 & \\
\hline $2390-2420$ & 2.613 & \\
\hline $2780-2810$ & 2.846 & \\
\hline $3200-3230$ & 2.661 & \\
\hline $3590-3620$ & 2.641 & \\
\hline $3980-4010$ & 2.799 & \\
\hline $4190-4220$ & 2.775 & $\begin{array}{l}\text { Argillite, dark gray, slaty, micaceous, + } \\
\text { graywacke? }\end{array}$ \\
\hline $4250-4280$ & 2.720 & $\begin{array}{l}\text { Serpentinite, green and brown, resinous, } \\
\text { sheared, pyroxene gabbro, felsic-Qtzose- } \\
\text { intrusive rocks }\end{array}$ \\
\hline $4370-4400$ & 2.669 & $\begin{array}{l}\text { Chrysotile bearing serpentinite, porphyritic } \\
\text { rhyolite, minor carbonate veins }\end{array}$ \\
\hline $4820-4850$ & 2.604 & Sheared serpentinite w/chrysotile, minor talc \\
\hline
\end{tabular}


Table 3. Occidental Geothermal, \#68-21, Sec. 21, T11N, R8W, Lake County, CA, elevation 3809 feet, total depth 7344 feet.

\begin{tabular}{|l|c|l|}
\hline $\begin{array}{l}\text { Sample } \\
\text { Identification }\end{array}$ & $\begin{array}{c}\text { Density } \\
\mathrm{g} / \mathrm{cm}^{3}\end{array}$ & Lithologies \\
\hline $100-150$ & 2.769 & Altered graywacke \\
\hline $400-450$ & 2.632 & Graywacke \\
\hline $800-850$ & 2.766 & Graywacke \\
\hline $1200-1250$ & 2.704 & $\begin{array}{l}\text { Graywacke with increasing interbedded argillite to } \\
45 \% \text { of sample }\end{array}$ \\
\hline $1600-1650$ & 2.812 & Graywacke w/var amounts interbedded Argillite \\
\hline $1960-2010$ & 2.973 & Greenstone \\
\hline $2400-2450$ & 2.920 & Altered greenstone and graywacke \\
\hline $2800-2850$ & 2.928 & $\begin{array}{l}\text { Greenstone w/5\% each of graywacke, argillite, } \\
\text { altered volcanic/greenstone }\end{array}$ \\
\hline & & $\begin{array}{l}\text { No samples available for density measurements in } \\
\text { this interval. Log shows mostly greenstone to 3570 } \\
\text { feet, then serpentinite }\end{array}$ \\
\hline $4000-4050$ & 2.826 & Serpentinite \\
\hline $4400-4450$ & 2.759 & Serpentinite \\
\hline $5000-5050$ & 3.006 & Graywacke \\
\hline $5500-5550$ & 3.013 & Melange: 45\% Grnstn, 35\% Argil, 20\% Grwke \\
\hline $5800-5850$ & 2.813 & $\begin{array}{l}\text { Melange: Graywacke 40-70\%, Arg 10-40\%, } \\
\text { Greenstone 10\%, Serp 5\% }\end{array}$ \\
\hline $6200-6250$ & 2.828 & Graywacke w/interbedded lenses of Argillite \\
\hline $6600-6650$ & 2.891 & Graywacke \\
\hline $7000-7050$ & 2.814 & Graywacke with 10-20\% Argillite \\
\hline $7300-7340$ & 2.878 & Graywacke with 20-40\% Argillite \\
\hline
\end{tabular}


Table 4a. Republic Boggs No. 77-1

\begin{tabular}{|l|l|l|}
\hline Sample & Density & Lithologies \\
\hline $2003-2004$ & 2.524 & Serpentine rich mudstone/sheared \\
\hline 2234 & 2.642 & \\
\hline
\end{tabular}

The samples in table $4 \mathrm{~b}$ are from large $>50 \mathrm{~g}$ samples. Most were measured very quickly since they appeared ready to fall apart in water, thus the weight in water measurements include some air and the resulting densities should be considered as minimums.

Table 4b. Republic Boggs No. 77-1

\begin{tabular}{|l|l|l|}
\hline Sample & Density & Lithologies and comments \\
\hline 1996 & 2.31 & \\
\hline 2000 & 2.22 & Mudstone (very fragile) \\
\hline 2029 & 2.31 & Fine grain wacke sandstone \\
\hline 2039 & 2.24 & Fine grain wacke sandstone (very fragile) \\
\hline 2070 & 2.42 & $\begin{array}{l}\text { Serp. pebble conglomerate, some clast, of vein } \\
\text { quartz (very fragile) }\end{array}$ \\
\hline 2184 & 2.29 & Sheared serpentinite \\
\hline 2309 & 2.65 & Sheared graywacke \\
\hline 2325 & 2.55 & \\
\hline 2339 & 2.59 & Sandy argillite \\
\hline 2386 & 2.59 & Black argillite \\
\hline 2427 & 2.57 & Black argillite \\
\hline
\end{tabular}


Table 5. Eureka-Magma-GRI-Getty, Kettenhoffen No1

Location is $150 \mathrm{feet} S$ and $125 \mathrm{ft} \mathrm{E}$ of the center of Section 28, T13N, R8W. Elevation 1836 feet.

\begin{tabular}{|c|c|c|}
\hline Sample Identification & $\begin{array}{l}\text { Density } \\
\mathrm{g} / \mathrm{cm}^{3}\end{array}$ & Lithologies \\
\hline $200-220 \& 240-260$ & 2.528 & $\begin{array}{l}\text { Basin deposits, sand, clay, conglomerate } \\
\text { rich in volcanic rock clasts (assorted } \\
\text { volcanic rock fragments) }\end{array}$ \\
\hline $500-520 \& 540-560$ & 2.592 & $\begin{array}{l}\text { Clear Lake Volcanics (CLV); biotite- } \\
\text { hornblende dacite }\end{array}$ \\
\hline $800-820 \& 840-860$ & 2.662 & CLV (andesite) \\
\hline $1100-1120 \& 1140-1160$ & 2.578 & \multirow[t]{4}{*}{ CLV } \\
\hline $1400-1420$ & 2.593 & \\
\hline $1700-1720 \& 1740-1760$ & 2.634 & \\
\hline $2100-2120 \& 2140-2160$ & 2.578 & \\
\hline$(2430)$ & & Top of Great Valley \\
\hline $2440-2460$ & 2.531 & $\begin{array}{l}\text { Great Valley (GV) sequence shale, } \\
\text { siltstone: and serpentinite, in part sheared } \\
\text { (medium dark gray shale interbedded } \\
\text { with minor siltstone) }\end{array}$ \\
\hline $2600-2620$ & 2.728 & $\begin{array}{l}\text { Fine grained micrograywacke intermixed } \\
\text { with sheared black argillite - } 60 \% \text {, talcose } \\
\text { sheared serpentinite }-30 \% \text {, white resinous } \\
\text { talc - 5\%, Clear Lake Volcanics possibly } \\
1-2 \%\end{array}$ \\
\hline $2700-2720 \& 2740-2760$ & 2.693 & $\begin{array}{l}\text { Scaly, sheared black argillite, trace of } \\
\text { green serpentinite, trace of Clear Lake } \\
\text { Volcanics (down hole?) }\end{array}$ \\
\hline $3000-3020 \& 3040-3060$ & 2.743 & $\begin{array}{l}\text { GV (dark gray shale with minor } \\
\text { interbedded siltstone) }\end{array}$ \\
\hline $3300-3320 \& 3340-3360$ & 2.757 & $\begin{array}{l}\text { GV (dark gray shale \& siltstone } \\
\text { interbedded serpentine cuttings } 10 \% \text { ) }\end{array}$ \\
\hline $3600-3620$ & 2.545 & GV (serpentine, shale and siltstone) \\
\hline $3800-3820$ & 2.587 & GV (serpentine sheared) \\
\hline
\end{tabular}

